\title{
Isolation of gene sets affected specifically by polyglutamine expression: implication of the TOR signaling pathway in neurodegeneration
}

\author{
B Nelson ${ }^{1,5}$, S Nishimura ${ }^{2}$, H Kanuka ${ }^{3}$, E Kuranaga ${ }^{3}$, M Inoue ${ }^{2}$, \\ G Hori $^{4}$, H Nakahara ${ }^{2}$ and M Miura ${ }^{*, 1,3}$ \\ 1 Laboratory for Cell Recovery Mechanisms, Brain Science Institute, RIKEN, 2-1 \\ Hirosawa, Wako, Saitama 351-0198, Japan \\ 2 Laboratory for Mathematical Neuroscience, Brain Science Institute, RIKEN, \\ 2-1, Hirosawa, Saitama 351-0198, Japan \\ ${ }^{3}$ Department of Genetics, Graduate School of Pharmaceutical Sciences, \\ University of Tokyo, 7-3-1 Hongo, Bunkyo-ku, Tokyo 113-0033, Japan \\ ${ }^{4}$ Laboratory for Advanced Brain Signal Processing, Brain Science Institute, \\ RIKEN, 2-1, Hirosawa, Saitama 351-0198, Japan \\ ${ }^{5}$ Current address: The Wellcome Trust Centre for Cell Biology, University of \\ Edinburgh, SBS, Institute of Cell Biology, Michael Swann Building, Mayfield \\ Rd, Edinburgh EH9 3JR, UK \\ * Corresponding author: M Miura, Department of Genetics, Graduate School of \\ Pharmaceutical Sciences, University of Tokyo, 7-3-1 Hongo, Bunkyo-ku, \\ Tokyo 113-0033, Japan. Tel: + 81-3-5841-4860; Fax: + 81-3-5841-4867; \\ E-mail: miura@mol.f.u-tokyo.ac.jp
}

Received 01.3.04; revised 02.2.05; accepted 22.2.05; published online 29.4.05 Edited by $\mathrm{H}$ Ichijo

\begin{abstract}
Transcriptional dysregulation as a result of sequestration of essential transcription factors into protein aggregates formed by polyglutamine (polyQ) expansions can lead to late-onset progressive neurodegeneration. DNA microarray analysis of Drosophila expressing polyQ in the compound eye over time revealed large numbers of transcriptional changes at the earliest stages of the disease including repression of the transient receptor potential calcium channels in a polyQ-induced cell death specific manner. While significant differences in expression profiles were found between the Drosophila compound eye and polyQsensitive neural cells, a number of possible key overlapping regulators were extracted. Among these, PDK1 was shown to act as a mediator for polyQ-toxicity, suggesting the involvement of the TOR pathway in polyQ-induced neurodegeneration.

Cell Death and Differentiation (2005) 12, 1115-1123.

doi:10.1038/sj.cdd.4401635; published online 29 April 2005
\end{abstract}

Keywords: polyglutamine expansion; neurodegeneration; DNA microarray; Drosophila

Abbreviations: polyQ, polyglutamine; Htt, Huntingtins

\section{Introduction}

The underlying mechanisms leading to polyglutamine (polyQ)-induced neurodegeneration of diseases such as
Huntington's disease and the spinocerebeller ataxias remain controversial. Expression of proteins containing an expansion of a CAG trinucleotide repeat leads to a clear accumulation of unfolded proteins in the form of aggregates appearing in diseased tissue; however, it is still uncertain whether these inclusion bodies cause cellular disruption or play a protective role. ${ }^{1-3} \mathrm{~A}$ further complication stems from tissue expression patterns of polyQ disease causing proteins; despite their ubiquitous expression, only postmitotic neurons are affected.

Models for polyQ-induced diseases, including Caenorhabditis elegans, Drosophila, and mice take advantage of easily amendable genetics to investigate neurodegeneration. ${ }^{4-9}$ From these studies, numerous insights have come to light including a role for molecular chaperones in delaying the onset of neurodegeneration. ${ }^{6,10}$ The neurodegeneration requires a functional translocon able to transport, as yet unknown, toxic protein(s) into the cytoplasm. ${ }^{11,12}$ Proteasome inhibition also likely contributes to the accumulation of toxic proteins. ${ }^{13,14}$

Another model to explain the mechanisms behind neurodegeneration suggests sequestration of essential proteins including transcription factors. ${ }^{15-18}$ Expanded polyQ-containing proteins have been shown to bind the transcriptional coactivator and acetyltransferase, CREB-binding protein (CBP) resulting in its depletion from the nucleus. ${ }^{15,19}$ In agreement with this model, both addition of histone deacetylases as well as overexpression of CBP have been shown to reduce polyQ pathology. ${ }^{20,21}$

DNA microarrays provide an excellent means by which to monitor global changes in gene expression and have been used successfully to study cell signaling events. ${ }^{22-24}$ Therefore, in order to examine possible transcriptional dysregulation during the progression of polyQ pathogenesis in Drosophila, polyQ-specific gene sets were isolated in this study. Identification of a gene set that is specifically affected by polyQ before the onset of neuronal degeneration is especially important because these genes must be early markers of disease progression as well as potential targets of the therapeutic drug. We could successfully isolate a common set of gene induction and repression before and after the onset of neuronal degeneration in a polyQ-induced cell death specific manner. These gene changes may be involved in polyQ-induced neural dysfunction that precedes the degeneration. In addition to measuring transcript levels in a late-onset neurodegeneration model of flies, we measured gene profiles of an in vitro model of polyQ-induced cell death (rapid cell death model) and through comparison of these profiles several potential regulators of polyQ-induced cell death were extracted. Among these, the PKD1/S6K pathway was shown to be required for the degeneration thereby implicating TOR signaling in polyQ-induced neurodegeneration. 


\section{Results}

\section{Isolation of polyglutamine-specific gene sets}

PolyQ-induced cell death is a complex process that cannot be blocked by caspase inhibition; however, firm connections between polyQ-induced disease progression and transcriptional dysregulation have been established. We therefore used DNA microarrays to monitor global changes in transcription in tissues expressing a polyQ expansion protein. Figure 1 shows a schematic of the experimental design enabling early transcriptional changes to be compared with those derived from late stages of neurodegeneration. The UAS-GAL4 system was used to express a 78 polyQ repeat expansion under control of the eye-specific promoter GMR. Under this system, neurons undergo a late-onset progressive degeneration leading to the deterioration of the eye without affecting fly viability (Figure 1). Total RNA was isolated from the heads of adult flies harvested at day 1 whereas GMR-GAL4 flies crossed to wild-type flies lacking the poly $Q$ protein were used as a control. Set at a $99 \%$ confidence level, by day 1 nearly 800 genes showed altered transcription including the heat shock family of proteins (Figure $2 \mathrm{a}$ ). Considering very little degeneration of eye neural tissue can be seen by day 1 (Figure 1), these transcripts represent putative indicators

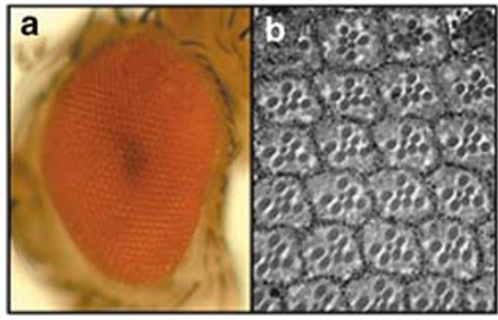

wild-type day 1

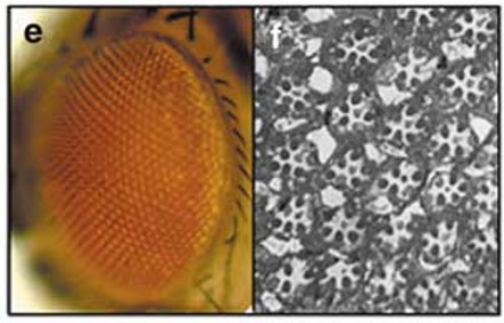

poly $Q$ day1

EGFP

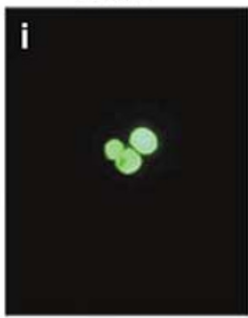

polyQ

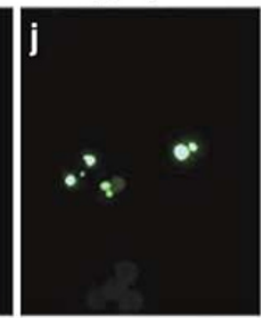

Non-Neural Cells

m

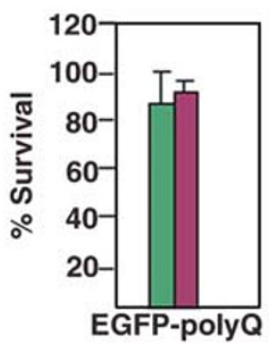

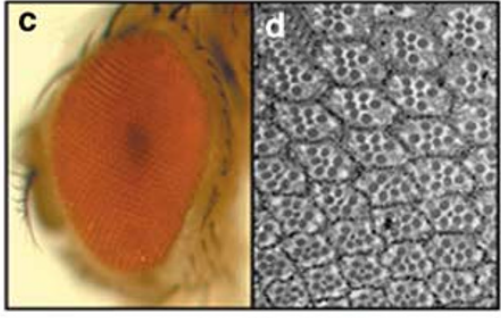

wild-type day 30

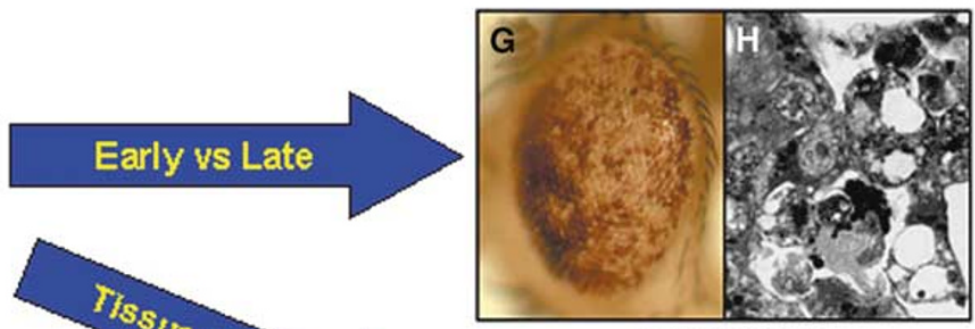

polyQ day30
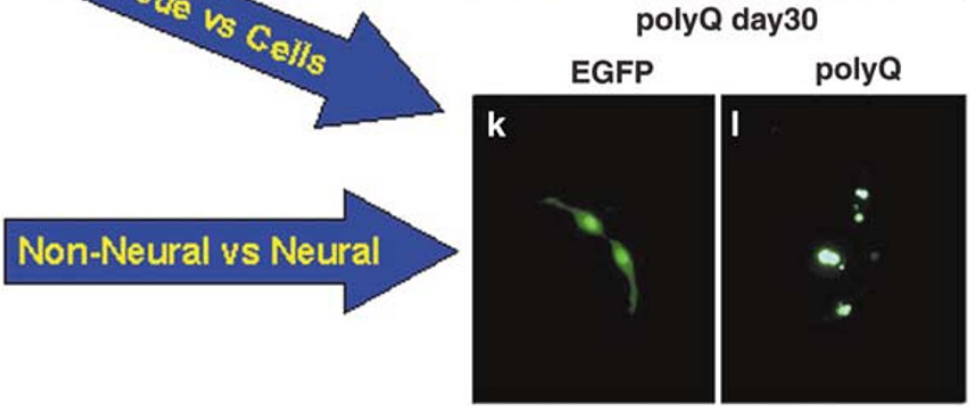

Neural Cells

n

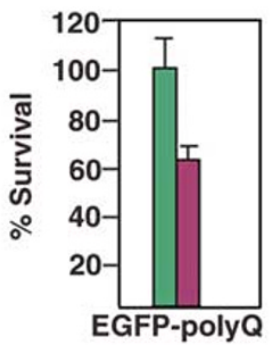

Figure 1 Schematic of experimental design to derive polyQ-specific transcripts. Briefly, comparison of expression profiles derived from day 1 flies (both wild-type and polyQ-expressing) to day 30 flies allowed early (day 1) versus late (day 30) gene changes to be elucidated. Similarly, expression profiles derived from neural and nonneural stable cell lines expressing EGFP and polyQ were used to compare neural and non-neural polyQ transcriptional changes. Overlap of tissue and cell line polyQspecific transcripts were then elucidated. (a, $\mathbf{c}, \mathbf{e}$, and $\mathbf{g}$ ) show the exterior of fly eyes. (b, $\mathbf{d}, \mathbf{f}$, and $\mathbf{h}$ ) show the interior of fly eyes. (i and $\mathbf{k}$ ) show EGFP-expressing cells while ( $\mathbf{j}$ and $\mathbf{I}$ ) show polyQ-expressing cells. ( $\mathbf{m}$ and $\mathbf{n}$ ) show cell death assays $24 \mathrm{~h}$ after heat shock 

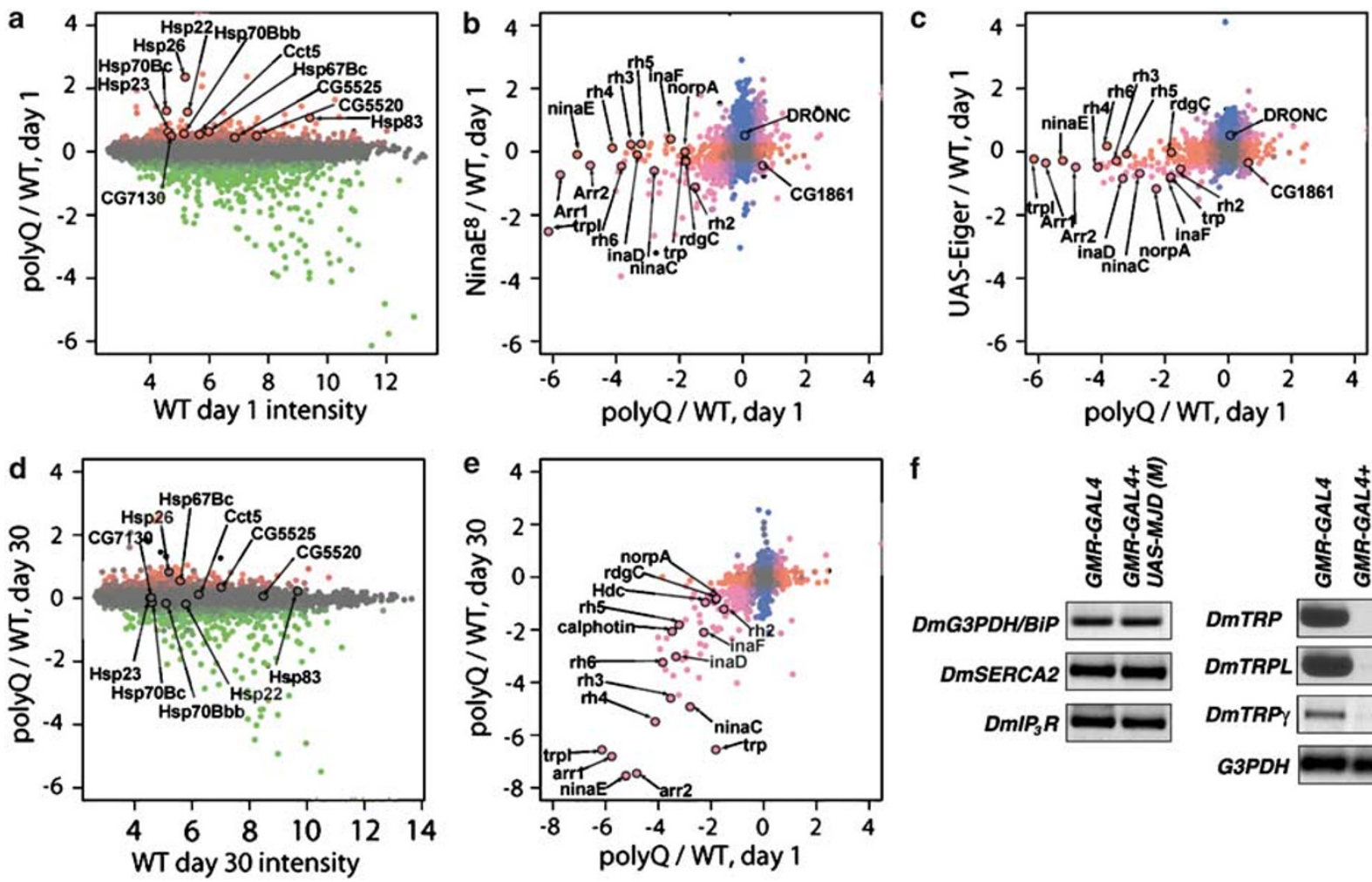

f
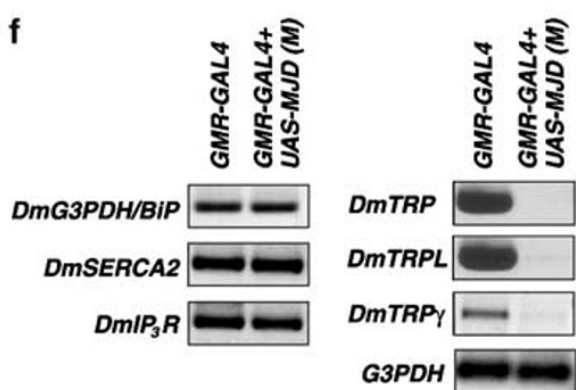

g

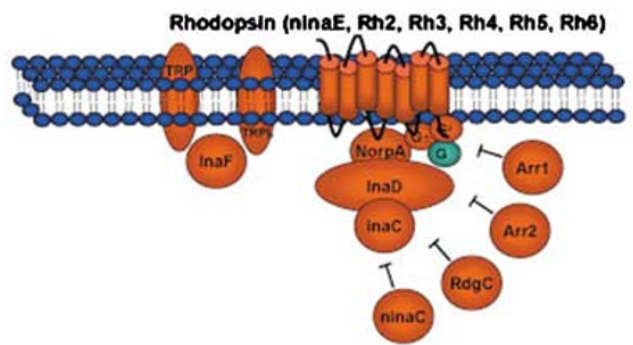

Figure 2 DNA microarray analysis of Drosophila heads expressing a 78 polyQ repeat in post mitotic neurons reveals widespread transcriptional changes. (a) Expression profile derived from polyQ-expressing flies compared to control flies both harvested at day 1 after eclosion. Data are displayed as intensity versus polyQexpressing/control ratio (log scale). Red dots represent gene induction, green dots gene repression. Color intensity of each spot represents the degree of confidence from gray $(P>0.1)$ to red/green $\left(P<10^{-5}\right)$. (b) Correlation plot of nina $E^{8}$ mutants (expression profile derived from control and nina $E^{8}$ homozygotes) and polyQexpressing flies (expression profile derived from control and polyQ-expressing flies) both harvested at day 1. Genes whose expression increased or decreased in both experiments are plotted as pink stars. Blue dots correspond to genes whose expression changed only in the transcriptional profile plotted on the $y$-axis whereas red dots correspond to genes whose expression changed only in the transcriptional profile plotted on the $x$-axis. Decreasing color intensity represents decreasing statistical significance with gray dots representing genes whose expression did not change significantly in either experiment at a $99 \%$ confidence level. (c) Correlation plot of eigerexpressing (expression profile derived from control and eiger-expressing flies) and polyQ-expressing flies (expression profile derived from control and polyQ-expressing flies) both harvested at day 1. (d) Expression profile derived from polyQ-expressing flies compared to control flies both harvested at day 30 after eclosion. Data are displayed as intensity versus polyQ-expressing/control ratio (log scale). (e) Correlation plot of day 1 (expression profile derived from control and polyQ expressing flies) and day 30 (expression profile derived from control and polyQ expressing flies) harvested flies. (f) Specific reduction of TRP channels in polyQ-expressing flies. The indicated genotypes of adult fly eyes were used for RT-PCR analysis. (g) Schematic of the inositide signaling phototransduction pathway in flies. Genes in red represent transcriptionally repressed genes in response to polyQ expression

of early disease state in polyQ-induced neurodegeneration in addition to containing possible key regulators of the disease. Furthermore, since less than half the tissue from which RNA was extracted expressed GAL4, these changes likely underestimate the actual transcriptional changes actually occurring. A complete list of all statistically significantly changed transcripts, in addition to raw data, from all experiments can be found at http://www.brain.riken.jp/labs/ $\mathrm{mns} /$ Drosophila/.
In order to ensure that altered transcription detected in polyQ-expressing flies was not merely a result of nonspecific reductions in eye tissue, polyQ-expressing fly profiles were compared to a polyQ-independent, caspase-dependent progressive degenerative mutant profile. NinaE corresponds to the G-protein coupled photoreceptor in the phototransduction pathway of Drosophila eyes; ninaE ${ }^{8}$ homozygotes contain very little rhodopsin thereby leading to a late-onset eye degeneration and large numbers of transcriptional changes 
(data not shown). By comparing profiles derived from ninaE $E^{8}$ homozygote heads with polyQ-expressing flies at day 1, a large overlap of gene transcription could be seen (Figure $2 b$ ). However, despite this overlap, a unique set of genes induced/ repressed specifically in polyQ-expressing flies could be identified. By comparing expression profiles derived from polyQ-expressing flies with polyQ-independent cell death activators, gene sets containing putative key regulators of polyQ-induced degeneration can be elucidated; here, genes in red belong to this set (Figure $2 b$, Supplementary information Table 1).

As another control, flies expressing a caspase-independent but nonprogressive, non-late-onset cell death activator were profiled. Eiger, a tumor necrosis factor (TNF) ligand, driven by a weak GMR-GAL4 line shows weak eye degeneration and large numbers of gene transcription changes by day $1^{25}$ (data not shown). Little overlap was seen between transcriptional profiles of Eiger-expressing and polyQ-expressing flies, further suggesting that many transcriptional changes in polyQ-expressing flies were a direct result of polyQ expression rather than mere loss of tissue (Figure 2c). Again, genes in red represent repressed/induced transcripts specific for polyQ-expression (Supplementary information Table 2).

\section{Polyglutamine-specific changes are not affected by ageing: reduction of TRP channels prior to the onset of degeneration}

In order to ascertain whether the aging process affected any polyQ-specific changes, polyQ-expressing flies were harvested at day 30 when easily discernible and large-scale measurable changes in transcription would be expected (Figure 1). Indeed, many transcripts showed increases and decreases; however, the number was reduced compared to day 1 to 483, the majority still repressed (Figure 2d). Interestingly, many of the heat shock proteins were no longer induced. Despite the increased degeneration seen at day 30 , a large overlap in gene expression was seen when transcriptional profiles were compared to day 1 (Figure 2e). Many of the genes found to change transcription specifically in response to polyQ expression were found in this overlapping group suggesting that aging does not affect polyQ-induced gene changes.

An interesting group of genes found repressed specifically during polyQ expression was the phosphoinositide signaling phototransduction pathway (Supplementary information Table 3). The phototransduction pathway in Drosophila is complexed into a signaling unit referred to as the signalplex comprised of a G-protein coupled receptor, Rhodopsin1 (Rh1/NinaE) leading to a canonical phosphoinositide cascade ${ }^{26}$ (Figure 2g). Among these genes, Rhodopsin (Rh) $1 /$ ninaE together with $R h 2,3,4,5$, and 6 , inaD (a scaffold for subcellular compartmentalization of the phototransduction machinery), ninaC (calmodulin binding protein), and norpA (GTPase activator), were all transcriptionally repressed. Genes associated with the inositide signaling phototransduction pathway, including the light-activated calcium channels, transient receptor potential (TRP), and TRPL, inaF, and the negative regulators, arr1, arr2, and $r d g C$ (seine/threonine phosphatase), were all transcriptionally downregulated. Inter- estingly, spinocerebellar ataxia type 1 (SCA1) model mice have shown a transcriptional reduction in the TRP ortholog, TRP3 suggesting a potential conserved mechanism for polyQ-induced neurodegeneration. ${ }^{27,28}$ To confirm the reduction of these genes, we performed semiquantitative RT-PCR and a dramatic reduction of trp, trpl and trp $\gamma$ was observed (Figure 2f).

Dysregulation of cellular $\mathrm{Ca}^{2+}$-homeostasis has been postulated to be a component of Huntington's disease and a polyQ-induced downregulation of other $\mathrm{Ca}^{2+}$-homeostasis and mobilization genes including inositol triphosphate receptor 1 (IP3R1) and sarcoplasmic endoplasmic reticulum calcium ATPase type 2 (SERCA2) in mouse models of Huntington's disease and SCA1 pathogenesis has been seen. $^{27-29}$ No endoplasmic reticulum stress could be observed as measured by BiP levels nor could any changes in the expression of SERCA2 and IP3R be seen by RT-PCR in Drosophila (Figure 2f), further suggesting the importance of trp reduction in polyQ-induced neurodegeneration.

\section{Different neural cells yield different polyglutamine- induced gene changes: involvement of PDK1/S6K as an early determinant of severity of degeneration}

In order to determine whether polyQ-induced gene changes occurred commonly in vitro between different types of neural cells, the first exon of human Huntingin (Htt) containing a 150glutamine expansion was fused to EGFP and driven by the heat shock promoter in both Drosophila embryonic derived Schneider's S2 cells and Drosophila neural BG2 cells. Stable clones were isolated and heat shocked for $1 \mathrm{~h}$ at $37^{\circ} \mathrm{C}$. Both cell types formed protein aggregates, however, only the neural BG2 cells showed measurable cell death $24 \mathrm{~h}$ after heat shock (Figure 1). Global transcriptional changes were monitored by DNA microarrays in both S2 and BG2 clones before and $24 \mathrm{~h}$ after heat shock. Stable S2 and BG2 clones expressing EGFP alone were simultaneously profiled. Sets of repressed and induced transcripts belonging specifically to neural BG2 cells can be seen in red (Figure 3a, Supplementary information Table 4).

When comparing the fly eye transcriptional profile with that derived from BG2 cells there is little overlap between gene sets (Figure 3b, Supplementary information Table 5). Unlike fly eyes, BG2 cell death is rapidly induced by polyQ expression (Figure 1) possibly accounting for the differences in gene sets. Furthermore, reaper was transcriptionally induced specifically in polyQ-expressing neural BG2 cells (and not in S2 cells) and may be responsible for the initiation of this rapid polyQ-induced cell death (Figure $3 a$ ).

Genes with commonly altered transcription in response to polyQ expression, both in fly eyes and neural cell lines, may act as key regulators of neurodegeneration. Table 1 lists polyQ-specific genes in common that are polyQ-specific; that is, not changed in nina $E^{8}$ mutants or Eiger-expressing flies. From this table, only nine transcripts were commonly transcriptionally induced including appl, encoding a beta amyloid protein precursor ortholog implicated in Alzheimer's disease (Figure 3b, Table 1 and Supplementary information Table 5). A total of 45 repressed transcripts were shared 

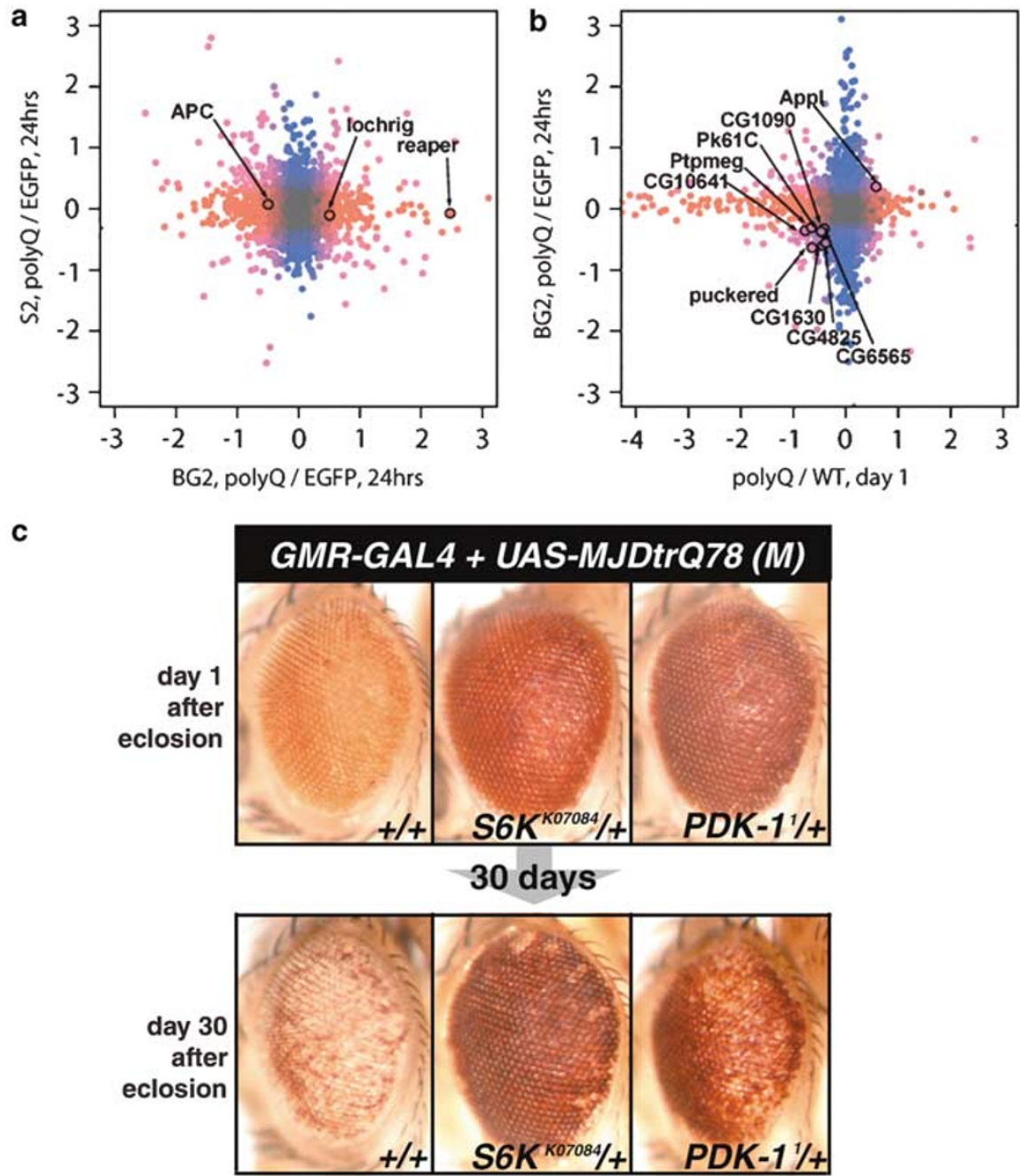

Figure 3 Downregulation of PDK1 and S6K alleviate neurodegeneration. (a) Correlation plot of non-neural S2 cells (expression profile derived from control EGFP and polyQ-expressing stable cells 24hrs after heat shock) and neural BG2 cells (expression profile derived from control EGFP and polyQ-expressing stable cells 24hrs after heat shock). Colors are as indicated in Figure 2. (b) Correlation plot of neural BG2 cells (expression profile derived from control EGFP and polyQ-expressing stable cells 24hrs after heat shock) and day 1 (expression profile derived from control and polyQ-expressing flies) harvested flies. (c) Eye morphology of wt, S6K heterozygoutes and $P D K-1$ heterozygoutes all expressing polyQ

among gene sets, many of which are uncharacterized. puckered, a JUN kinase phosphatase and a JNK signal target gene, ${ }^{30}$ and ptpmeg, a protein tyrosine phosphatase, were transcriptionally downregulated in both gene sets. Phospholipid bilayer synthesis may be affected as CG4825, a putative CDP-diacylglycerol-serine-O-phosphatidyltransferase, and CG6565 encoding a phosphatidylcholine transporter were both transcriptionally repressed. Lastly, both calcium regulation and inositol signaling were further implicated as CG1090, CG10641, CG1630, and Pk61C (dPDK-1) were all transcriptionally downregulated.

We tested for genetic interactions with available mutants and found that reduction of Drosophila phosphoinositidedependent protein kinase-1 (dPDK1) significantly suppressed polyQ-induced eye degeneration (Figure $3 \mathrm{c}$ ). PDK1 acts as an activator of the AGC family protein kinases, including AKT, p70 ribosomal S6 kinase (S6K), serum- and glucocorticoideinduced protein kinase (SGK) and protein kinase $C(P K C)$.
Reduction of the PDK1 effecter, S6K, also suppressed the progression of polyQ-induced eye degeneration (Figure 3c). The finding of common transcriptional changes in both cell lines and flies expressing polyQ suggests PDK1 and S6K are affected directly by polyQ expression and determining their role in polyQ-induced neurodegeneration may shed light on mechanisms underlying the degeneration.

\section{Discussion}

Compare plots provide an easy, attractive method of displaying large amounts of data generated from genomic experiments and through such manipulation of data sets many potential regulators of polyQ-induced neurodegeneration were identified. Several genes whose products have been implicated in cell death were found to have altered transcription specifically in response to polyQ expression including 
Table 1 Genes with altered expession in both flies and cell line specifically in response to polyglutamine expression

\begin{tabular}{|c|c|c|c|c|}
\hline \multirow[b]{2}{*}{ Rank 1} & \multicolumn{2}{|c|}{ Fold change } & \multirow[b]{2}{*}{ Gene title } & \multirow[b]{2}{*}{ Function } \\
\hline & Flies & Cells & & \\
\hline \multicolumn{5}{|c|}{ Induced } \\
\hline 1 & 1.28 & 3.56 & CG3996 & \\
\hline 2 & 1.27 & 2.13 & CGI 8547 & \\
\hline 3 & 1.29 & 1.75 & CG31847 & \\
\hline 4 & 1.48 & 1.42 & CGI 0353 & \\
\hline 5 & 1.49 & 1.29 & Appl beta amyloid protein precursor-like & Neurogenesis \\
\hline 6 & 1.41 & 1.26 & CG32611 & Nucleic acid binding \\
\hline 7 & 1.33 & 1.26 & CG2076 & \\
\hline 8 & 1.36 & 1.20 & mus209 mutagen-sensitive 209 & Nucleotide-excision repair \\
\hline 9 & 1.25 & 1.27 & CGI 5097 & Actin binding \\
\hline \multicolumn{5}{|c|}{ Repressed } \\
\hline 1 & 1.46 & 3.94 & CG6113 & Triacylglycerol lipase activity \\
\hline 2 & 3.69 & 1.46 & CG7220 & Ubiquitin conjugating enzyme activity \\
\hline 3 & 1.91 & 1.81 & CG5287 & Catalytic activity \\
\hline 4 & 1.80 & 1.96 & CAP & Focal adhesion \\
\hline 5 & 1.74 & 1.83 & CG8468 & Monocarboxylate porter activity \\
\hline 6 & 1.44 & 1.82 & CGI 3784 & Signal transduction \\
\hline 7 & 1.56 & 1.56 & puc puckered & JNK phosphatase \\
\hline 8 & 1.50 & 1.56 & CGI 0960 & Glucose transporter activity \\
\hline 9 & 1.65 & 1.48 & didum dilute class unconventional myosin & Motor activity \\
\hline 10 & 1.96 & 1.31 & CG10311 & Transport \\
\hline 11 & 1.47 & 1.60 & CG4196 & \\
\hline 12 & 1.53 & 1.48 & CG18316 & \\
\hline 13 & 1.48 & 1.55 & Kap-alphal karyopherin alpha 1 & Protein-nucleus import \\
\hline 14 & 1.42 & 1.47 & CG32428 & \\
\hline 15 & 1.83 & 1.31 & Dnzl DNZDHHC/NEW1 zinc-finger protein 11 & \\
\hline 16 & 1.95 & 1.43 & CG31116 & Voltage-gated chloride channel activity \\
\hline 17 & 1.29 & 1.46 & CG15917 & \\
\hline 18 & 2.55 & 1.27 & Fatp Fatty acid (long chain) transport protein & Catalytic activity \\
\hline 19 & 1.70 & 1.26 & vri vrille & RNA polymerase II transcription factor activity \\
\hline 20 & 1.38 & 1.44 & CG1939 & ATP binding \\
\hline 21 & 1.35 & 1.52 & IP3K2 Inositol 1,4,5-triphosphate kinase 2 & 1D-myo-inositol-trisphosphate 3-kinase activity \\
\hline 22 & 1.30 & 1.48 & CG4825 & Phosphatidylserine biosynthesis \\
\hline 23 & 1.58 & 1.27 & CGI 2292 & \\
\hline 24 & 1.57 & 1.24 & Ptpmeg & Intracellular signaling cascade \\
\hline 25 & 1.46 & 1.41 & crq croquemort & Scavenger receptor activity \\
\hline 26 & 1.36 & 1.32 & Olf186-F & NOT serine-type endopeptidase activity \\
\hline 27 & 1.38 & 1.30 & PDK-1 & Positive regulation of cell growth \\
\hline 28 & 1.36 & 1.31 & CG6565 & Phosphatidylcholine transporter activity \\
\hline 29 & 1.41 & 1.54 & Arc70 & Transcription initiation from Pol II promoter \\
\hline 30 & 1.71 & 1.28 & CGI 0641 & Calcium ion binding \\
\hline 31 & 1.46 & 1.34 & $\mathrm{l}(2) \mathrm{k} 09913$ & \\
\hline 32 & 1.25 & 1.34 & CGI 1857 & Vesicle-mediated transport \\
\hline 33 & 1.41 & 1.36 & Nrvl Nervana 1 & Potassium ion transport \\
\hline 34 & 1.52 & 1.34 & ctp cut up & Microtubule-based movement \\
\hline 35 & 1.37 & 1.45 & lace lace & Serine C-palmitoyltransferase activity \\
\hline 36 & 1.34 & 1.27 & CGI 1537 & Sugar porter activity \\
\hline 37 & 1.33 & 1.25 & CGI 090 & Calcium, potassium:sodium antiporter activity \\
\hline 38 & 1.50 & 1.31 & Nc73EF Neural conserved at 73KF & Oxidoreductase activity \\
\hline 39 & 1.28 & 1.25 & alphaTub84D Tubulin at 84D & Microtubule-based process \\
\hline 40 & 1.29 & 1.35 & EG.86E4.2 & \\
\hline 41 & 1.28 & 1.28 & Pfrx fi-phosphofructo-2-kinase & Catalytic activity \\
\hline 42 & 1.26 & 1.26 & CG10338 & \\
\hline 43 & 1.48 & 1.26 & nes nessy & \\
\hline 44 & 1.39 & 1.25 & CG8057 antisense construct a of Pan & SNF1 A/AMP-activated protein kinase activity \\
\hline
\end{tabular}

Ranking is based on joint probability (multiplicaton of) $P$-values for flies (polyQAVT day 1 comparison) and cell line (polyQ/EGFP BG2 cell comparison)

CG1861, calphotin, Hdc, Apc, and lochrig. CG1861 is highly similar to $\mathrm{dX} 11 \mathrm{~L}$ especially in the PDZ-domain region; overexpression of this region of $\mathrm{dX} 11 \mathrm{~L}$ is sufficient to induce eye degeneration. ${ }^{31}$ Calphotin is a calcium binding protein crucial to both rhabdomere development and photoreceptor cell survival. ${ }^{32} \mathrm{Hdc}$ functions as a major neurotransmitter in photoreceptor cells and $\mathrm{Hdc}$ mutants are blind. ${ }^{33} A p c$ is an ortholog of the adenomatous polyposis coli $(A P C)$ tumor suppressor gene whose disruption leads to retinal degenera- tion. $^{34}$ lochrig mutants display neurodegeneration that is enhanced by mutation to the amyloid precursor protein-like (App) involved in Alzheimer's disease. ${ }^{35}$ Many such genes show small but statistically significant changes in expression in response to polyQ expression and it is perhaps an accumulation of such changes that leads to the slow progressive, late-onset neurodegeneration that is the hallmark of the polyQ family of diseases. Without such data representation it may not have been apparent that such genes 
displayed transcriptional induction/repression specifically in response to polyQ expression.

One key biological mechanism may have been uncovered when polyQ-expressing flies undergoing neurodegeneration revealed the transcriptional repression of the TRP channels. In fact, mTRP3 was found repressed in polyQ-expressing mice further suggesting a conserved mechanism of neurodegeneration. ${ }^{27,28}$ Furthermore, due to the absence of significant neurodegeneration on day 1 it appears that TRPs may serve as markers for the risk of neurodegeneration prior to the onset of the disease.

Phosphoinositide signaling has been implicated in a variety of cellular activities including cell survival, cell growth, and autophagy ${ }^{36-38}$ and its downregulation in response to polyQ expression in flies may represent a general mechanism of neurodegeneration. One of the interesting findings of our microarray analysis combined with genetic interaction is the involvement of PDK1/S6K in polyQ-induced neurodegeneration. Recently, it has been shown that mTOR is sequestered in polyQ aggregates thereby inhibiting its kinase activity and inducing autophagy. ${ }^{39}$ Furthermore, specific inhibition of mTOR by rapamycin attenuates polyQ-induced aggregation and cell death. Since PDK1/S6K is involved in the TOR pathway, the observed reduction of PDK1 in both polyQexpressing BG2 cells and day 1 fly eyes may represent a protective cellular response against polyQ toxicity through enhancement of autophagy by inhibition of the TOR pathway.

\section{Materials and Methods}

\section{Fly stocks}

All general fly stocks and GAL4 lines were obtained from Drosophila stock centers. white ${ }^{1118}$ and GMR-GAL4 were used as a wild-type line for all experiments. The UAS-MJDtr-Q78 flies were a gift from $\mathrm{N}$ Bonini (University of Pennsylvania). The UAS-eiger flies were constructed at the same time and same manner as those found in Igaki et al. ${ }^{25} P D K-1^{1}$ was a gift from J Chung. ${ }^{40} S 6 K^{K 07084}$ was a gift from G Thomas. ${ }^{41}$ ninaE ${ }^{8}$ homozygote flies were received from the Bloomington Stock Center. Fly cultures and crosses were performed at $22^{\circ} \mathrm{C}$ following standard protocols. All flies were stored in bottles containing approximately 50 flies and media was changed once every 3 days. Fly histology was performed as described. ${ }^{11}$

\section{Cell culture}

Drosophila S2 and BG2 cells were cultured and transfected as reported. ${ }^{11}$ To make stable cell lines (both S2 and BG2), cells were cotransfected with $4.5 \mathrm{ng}$ of either $p$ CaspeR-hs-EGFP or pCaspeR-hs-tNhtt-Q150-EGFP and $0.5 \mathrm{ng}$ of a geneticin-expressing plasmid ( $p A c t 5 c o-N e o m y c i n)$ in a six-well plate. Cultures were grown in the presence of geneticin $(1 \mathrm{mg} / \mathrm{ml})$ and diluted in a $10 \mathrm{~cm}$ dish. Individual cells were allowed to form colonies that were picked (40 of each), grown, and tested for EGFP expression. Cell death assays were performed as described. ${ }^{11}$

\section{Plasmid construction}

The pCaspeR-hs-tNhtt-Q150-EGFP, pCaspeR-hs-EGFP, and pAct5coNeomycin (a gift from $S$ Nagata) plasmids have been described. ${ }^{11,42,43}$ Original tNhtt constructs were provided by N Nukina. ${ }^{44}$

\section{RT-PCR}

The indicated genotypes of adult flies were used for RT-PCR analysis (26 cycles). The compound eyes of anesthetized adult flies were dissected carefully, and the total RNA of the compound eye of adult flies was extracted by TRIzol reagent (GIBCO). To detect mRNA expression, $1 \mu \mathrm{g}$ of total RNA was reverse-transcribed in a total volume of $30 \mu \mathrm{l}$, and $1 \mu \mathrm{l}$ of the reaction mixture was used to amplify the transcripts by step-down PCR with ExTaq (Takara) using specific primer sets in a total volume of $25 \mu \mathrm{l}$. The following primer sets were used: Drosophila BiP/GRP78, 5'-CAT GGT TCT GGG CAA GAT GAA GGA AAC CGC- $3^{\prime}$ and $5^{\prime}$-CAC AAT TTC GTG CAC GTC CTT CTT GTT CAT-3'; Drosophila SERCA2 (Ca-P60A) ${ }^{45} 5^{\prime}$ AAG TCC TAC TCG GGT CGT GAA TTC GAC GAC-3' and $5^{\prime}$-AGC CAT GGT CAT CGC ATG GGG GTC GCT GAA-3'; Drosophila IP3R ${ }^{46} 5^{\prime}$-CTT CGA TTC ATC TTT TTA CTA GGG CCG GAG-3' and 5'-GGC GAA GGT GTC GAT GAT GAC ACC AAA TAT-3'; Drosophila TRP $\gamma^{47}, 5^{\prime}$-ATC GAT TTG GAC AGC TTT GAG TTG GAT GGA-3' and 5'-GTC CAA CGA TGT GGA GAA CTC GGC AAC TGG-3'; Drosophila TRP, 5'-GAC CAG GAG AAG GCT TGT ACC ATC TGG CGA-3' and $5^{\prime}$ - GAC GAA GCC GAT TTG GAA GTC CTT AAG GAT-3'; Drosophila TRPL, 5' - CTG AAC CAA CTG CTC TGG TAC TTT GCC GCC-3' and 5'- GTT GGC CGA GGA AAC ATC CAT TCC GCT GTT-3'. The equality of the amount of CDNA samples used for PCR was verified by PCR using the primer set for Drosophila G3PDH. The primer sequences used for the detection of Drosophila G3PDH have been described. ${ }^{48,43}$

\section{DNA microarrays}

Experiments, both fly and cell line, were repeated independently at least two times. Flies were anesthetized with $\mathrm{CO}_{2}$ and immediately flash frozen in liquid nitrogen. The heads of at least 500 flies for each experiment were isolated using mesh wire, and total RNA was extracted using Trizol and purified using Qiagen Rneasy Kits. Flies were collected at the same time each day to avoid fluctuations in circadian rhythms. Stable cell lines (both S2 and BG2) were seeded in $10 \mathrm{~cm}$ dishes at a density of $2 \times 10^{5}$ cells $/ \mathrm{ml}$ and left overnight at $25^{\circ} \mathrm{C}$. At the appropriate time point, media was aspirated and Trizol added directly to the dish, cells were scraped off and lysed with a fine-gauge syringe. Purified total RNA (15 $\mu \mathrm{g})$ was used to prepare biotinylated probes using standard Affymetrix protocols. Probes were hybridized to Affymetrix arrays using standard protocols.

\section{DNA microarray analysis}

Expression values were obtained using the Affy package ${ }^{49}$ of Bioconductor software available from http://www.bioconductor.org/. The parameters for the Affy package were set at default. Affy package outputs the referred expression measure as log-scale robust multi-array analysis (RMA). Values from RMA are set at log two therefore a difference of 1.0 is equal to a two-fold change. The comparisons of two groups were done using a Bayesian $t$-test approach, ${ }^{50}$ considered more robust than normal $t$-tests in small sampling. The $\mathrm{R}$ program scripts for the statistical calculation are downloadable from http://www.brain.riken.jp/labs/mns/Drosophila/.

\section{Acknowledgements}

We are grateful to T Nishimura, P Jorgensen, and G Cagney for comments on the manuscript, $\mathrm{N}$ Itoh for technical assistance and to the Bloomington Stock Center for fly stocks. We are also grateful to N Nukina, S Nagata, T Igaki, N Bonini, J Chung and G Thomas for materials. This work was supported in part by grants from the Japanese Ministry of Education, 
Science, Sports, Culture and Technology to MM, HN and SN. This work was also supported in part by a RIKEN Bioarchitect Research Grant to MM. HK is a research fellow of the Special Postdoctoral Researchers Program, RIKEN.

\section{References}

1. Sherman MY and Goldberg AL (2001) Cellular defenses against unfolded proteins: a cell biologist thinks about neurodegenerative diseases. Neuron 29: 15-32

2. Saudou F, Finkbeiner S, Devys D and Greenberg ME (1998) Huntingtin acts in the nucleus to induce apoptosis but death does not correlate with the formation of intranuclear inclusions. Cell 95: 55-66

3. Klement IA, Skinner PJ, Kaytor MD, Yi H, Hersch SM, Clark HB, Zoghbi HY and Orr HT (1998) Ataxin-1 nuclear localization and aggregation: role in polyglutamine-induced disease in SCA1 transgenic mice. Cell 95: 41-53

4. Fortini ME and Bonini NM (2000) Modeling human neurodegenerative diseases in Drosophila: on a wing and a prayer. Trends Genet. 16: 161-167

5. Faber PW, Alter JR, MacDonald ME and Hart AC (1999) Polyglutaminemediated dysfunction and apoptotic death of a Caenorhabditis elegans sensory neuron. Proc. Natl. Acad. Sci. USA 96: 179-184

6. Kazemi-Esfarjani $P$ and Benzer $S$ (2000) Genetic suppression of polyglutamine toxicity in Drosophila. Science 287: 1837-1840

7. Warrick JM, Paulson HL, Gray-Board GL, Bui QT, Fischbeck KH, Pittman RN and Bonini NM (1998) Expanded polyglutamine protein forms nuclear inclusions and causes neural degeneration in Drosophila. Cell 93: 939-949

8. Jackson GR, Salecker I, Dong X, Yao X, Arnheim N, Faber PW, MacDonald ME and Zipursky SL (1998) Polyglutamine-expanded human huntingtin transgenes induce degeneration of Drosophila photoreceptor neurons. Neuron 21: 633-642

9. Schilling G, Wood JD, Duan K, Slunt HH, Gonzales V, Yamada M, Cooper JK, Margolis RL, Jenkins NA, Copeland NG, Takahashi H, Tsuji S, Price DL, Borchelt DR and Ross CA (1999) Nuclear accumulation of truncated atrophin-1 fragments in a transgenic mouse model of DRPLA. Neuron 24: 275-286

10. Hsu AL, Murphy CT and Kenyon C (2003) Regulation of aging and age-related disease by DAF-16 and heat-shock factor. Science 300: 1142-1145

11. Kanuka $H$, Kuranaga $E$, Hiratou $T$, Igaki $T$, Nelson B, Okano $H$ and Miura $M$ (2003) Cytosol-endoplasmic reticulum interplay by Sec61 $\alpha$ translocon in polyglutamine-mediated neurotoxicity in Drosophila. Proc. Natl. Acad. Sci. USA 100: $11723-11728$

12. Higashiyama $\mathrm{H}$, Hirose $F$, Yamaguchi M, Inoue $Y H$, Fujikake N, Matsukage $A$ and Kakizuka A (2002) Identification of ter94, Drosophila VCP, as a modulator of polyglutamine-induced neurodegeneration. Cell Death Differ. 9: 264-273

13. Bence NF, Sampat RM and Kopito RR (2001) Impairment of the ubiquitinproteasome system by protein aggregation. Science 292: 1552-1555

14. Jana NR, Zemskov EA, Wang G and Nukina N (2001) Altered proteasomal function due to the expression of polyglutamine-expanded truncated $\mathrm{N}$-terminal huntingtin induces apoptosis by caspase activation through mitochondrial cytochrome c release. Hum. Mol. Genet. 10: 1049-1059

15. McCampbell A, Taylor JP, Taye AA, Robitschek J, Li M, Walcott J, Merry D, Chai Y, Paulson H, Sobue G and Fischbeck KH (2000) CREB-binding protein sequestration by expanded polyglutamine. Hum. Mol. Genet. 9: 2197-2202

16. Nucifora Jr. FC, Sasaki M, Peters MF, Huang H, Cooper JK, Yamada M, Takahashi H, Tsuji S, Troncoso J, Dawson VL, Dawson TM and Ross CA (2001) Interference by huntingtin and atrophin-1 with cbp-mediated transcription leading to cellular toxicity. Science 291: 2423-2428

17. Dunah AW, Jeong H, Griffin A, Kim YM, Standaert DG, Hersch SM, Mouradian $\mathrm{MM}$, Young $A B$, Tanese $N$ and Krainc D (2002) Sp1 and TAFII130 transcriptional activity disrupted in early Huntington's disease. Science 296: 2238-2243

18. Shimohata T, Nakajima T, Yamada M, Uchida C, Onodera O, Naruse S, Kimura T, Koide R, Nozaki K, Sano Y, Ishiguro H, Sakoe K, Ooshima T, Sato A, Ikeuchi T, Oyake M, Sato T, Aoyagi Y, Hozumi I, Nagatsu T, Takiyama Y, Nishizawa M, Goto J, Kanazawa I, Davidson I, Tanese N, Takahashi H and Tsuji S (2000) Expanded polyglutamine stretches interact with TAFII130, interfering with CREB-dependent transcription. Nat. Genet. 26: 29-36
19. Chai Y, Shao J, Miller VM, Williams A and Paulson HL (2002) Live-cell imaging reveals divergent intracellular dynamics of polyglutamine disease proteins and supports a sequestration model of pathogenesis. Proc. Natl. Acad. Sci. USA 99: 9310-9315

20. Taylor JP, Taye AA, Campbell C, Kazemi-Esfarjani P, Fischbeck KH and Min KT (2003) Aberrant histone acetylation, altered transcription, and retinal degeneration in a Drosophila model of polyglutamine disease are rescued by CREB-binding protein. Genes Dev. 17: 1463-1468

21. Steffan JS, Bodai L, Pallos J, Poelman M, McCampbell A, Apostol BL, Kazantsev A, Schmidt E, Zhu YZ, Greenwald M, Kurokawa R, Housman DE, Jackson GR, Marsh JL and Thompson LM (2001) Histone deacetylase inhibitors arrest polyglutamine-dependent neurodegeneration in Drosophila. Nature 413: 739-743

22. Roberts CJ, Nelson B, Marton MJ, Stoughton R, Meyer MR, Bennett $\mathrm{HA}, \mathrm{He}$ YD, Dai H, Walker WL, Hughes TR, Tyers M, Boone C and Friend SH (2000) Signaling and circuitry of multiple MAPK pathways revealed by a matrix of global gene expression profiles. Science 287: 873-880

23. DeRisi JL, lyer VR and Brown PO (1997) Exploring the metabolic and genetic control of gene expression on a genomic scale. Science 278: 680-686

24. Michaut L, Flister S, Neeb M, White KP, Certa U and Gehring WJ (2003) Analysis of the eye developmental pathway in Drosophila using DNA microarrays. Proc. Natl. Acad. Sci. USA 100: 4024-4029

25. Igaki $\mathrm{T}$, Kanda $\mathrm{H}$, Yamamoto-Goto $\mathrm{Y}$, Kanuka $\mathrm{H}$, Kuranaga $\mathrm{E}$, Aigaki $\mathrm{T}$ and Miura M (2002) Eiger, a TNF superfamily ligand that triggers the Drosophila JNK pathway. EMBO J. 21: 3009-3018

26. Montell C (1999) Visual transduction in Drosophila. Annu. Rev. Cell Dev. Biol. 15: $231-268$

27. Luthi-Carter R, Strand A, Peters NL, Solano SM, Hollingsworth ZR, Menon AS, Frey AS, Spektor BS, Penney EB, Schilling G, Ross CA, Borchelt DR, Tapscott SJ, Young AB, Cha JH and Olson JM (2000) Decreased expression of striatal signaling genes in a mouse model of Huntington's disease. Hum. Mol. Genet. 9: $1259-1271$

28. Lin X, Antalffy B, Kang D, Orr HT and Zoghbi HY (2000) Polyglutamine expansion down-regulates specific neuronal genes before pathologic changes in SCA1. Nat. Neurosci. 3: 157-163

29. Hodgson JG, Agopyan N, Gutekunst CA, Leavitt BR, LePiane F, Singaraja R, Smith DJ, Bissada N, McCutcheon K, Nasir J, Jamot L, Li XJ, Stevens ME, Rosemond E, Roder JC, Phillips AG, Rubin EM, Hersch SM and Hayden MR (1999) A YAC mouse model for Huntington's disease with full-length mutant huntingtin, cytoplasmic toxicity, and selective striatal neurodegeneration. Neuron 23: 181-192

30. Adachi-Yamada T, Fujimura-Kamada K, Nishida Y and Matsumoto K (1999) Distortion of proximodistal information causes JNK-dependent apoptosis in Drosophila wing. Nature 400: 166-169

31. Hase M, Yagi Y, Taru H, Tomita S, Sumioka A, Hori K, Miyamoto K, Sasamura $T$, Nakamura M, Matsuno K and Suzuki T (2002) Expression and characterization of the Drosophila X11-like/Mint protein during neural development. J. Neurochem. 81: 1223-1232

32. Yang $Y$ and Balinger D (1994) Mutations in calphotin, the gene encoding a Drosophila photoreceptor cell-specific calcium-binding protein, reveal roles in cellular morphogenesis and survival. Genetics 138: 413-421

33. Melzig J, Buchner S, Wiebel F, Wolf R, Burg M, Pak WL and Buchner E (1996) Genetic depletion of histamine from the nervous system of Drosophila eliminates specific visual and mechanosensory behavior. J. Comp. Physiol. 179: 763-773

34. Ahmed Y, Hayashi S, Levine A and Wieschaus E (1998) Regulation of armadillo by a Drosophila APC inhibits neuronal apoptosis during retinal development. Cell 93: 1171-1182

35. Tschape JA, Hammerschmied C, Muhlig-Versen M, Athenstaedt K, Daum G and Kretzschmar D (2002) The neurodegeneration mutant lochrig interferes with cholesterol homeostasis and Appl processing. EMBO J. 21: 6367-6376

36. Oldham S and Hafen E (2002) Insulin/IGF and target of rapamycin signaling: a TOR de force in growth control. Trends Cell Biol. 13: 79-85

37. Bjornsti M-A and Houghton PJ (2004) The TOR pathway: a target for cancer therapy. Nat. Rev. Cancer 4: 335-348

38. Gozuacik D and Kimchi A (2004) Autophagy as a cell death and tumor suppressor mechanism. Oncogene 23: 2891-2906

39. Ravikumar B, Vacher C, Berger Z, Davies JE, Luo S, Oroz LG, Scaravilli F, Easton DF, Duden R, O'Kane CJ and Rubinsztein DC (2004) Inhibition of 
mTOR induces autophagy and reduces toxicity of polyglutamine expansions in fly and mouse models of Huntington disease. Nat. Genet. 36: 585-595

40. Cho KS, Lee JH, Kim S, Kim D, Koh H, Lee J, Kim C, Kim J and Chung J (2001) Drosophila phosphoinositide-dependent kinase-1 regulates apoptosis and growth via the phosphoinositide 3-kinase-dependent signaling pathway. Proc. Natl. Acad. Sci. USA 98: 6144-6149

41. Montagne J, Stewart MJ, Stocker H, Hafen E, Kozma SC and Thomas G (1999) Drosophila S6 kinase: a regulator of cell size. Science 285: 2126-2129

42. Kondo T, Yokokura T and Nagata S. (1997) Activation of distinct caspase-like proteases by Fas and reaper in Drosophila cells. Proc. Natl. Acad. Sci. USA 94: 11951-11956

43. Kanuka H, Hisahara S, Sawamoto K, Shoji S, Okano H and Miura M (1999) Proapoptotic activity of Caenorhabditis elegans CED-4 protein in Drosophila: implicated mechanisms for caspase activation. Proc. Natl. Acad. Sci. USA 96: 145-150

44. Wang GH, Mitsui K, Kotliarova S, Yamashita A, Nagao Y, Tokuhiro S, Iwatsubo T, Kanazawa I and Nukina N (1999) Caspase activation during apoptotic cell death induced by expanded polyglutamine in N2a cells. Neuroreport 10 : 2435-2438
45. Magyar A and Varadi A (1990) Molecular cloning and chromosomal localization of a sarco/endoplasmic reticulum-type Ca2(+)-ATPase of Drosophila melanogaster. Biochem. Biophys. Res. Commun. 173: 872-877

46. Yoshikawa S, Tanimura T, Miyawaki A, Nakamura M, Yuzaki M, Furuichi T and Mikoshiba K (1992) Molecular cloning and characterization of the inositol 1,4,5-trisphosphate receptor in Drosophila melanogaster. J. Biol. Chem. 267: 16613-16619

47. Xu XZ, Chien F, Butler A, Salkoff L and Montell C (2000) TRP $\gamma$, a Drosophila TRP-related subunit, forms a regulated cation channel with TRPL. Neuron 26 : 647-657

48. Kanuka H, Sawamoto K, Inohara N, Matsuno K, Okano H and Miura M (1999) Control of the cell death pathway by Dapaf-1, a Drosophila Apaf-1/CED-4related caspase activator. Mol. Cell 4: 757-769

49. Irizarry RA, Bolstad BM, Collin F, Cope LM, Hobbs B and Speed TP (2003) Summaries of Affymetrix GeneChip probe level data. Nucleic Acids Res. 31: e15

50. Baldi $P$ and Long AD (2001) A Bayesian framework for the analysis of microarray expression data: regularized t-test and statistical inferences of gene changes. Bioinformatics 17: 509-519

Supplementary Information accompanies the paper on Cell Death and Differentiation website (http://www.nature.com/cdd) 